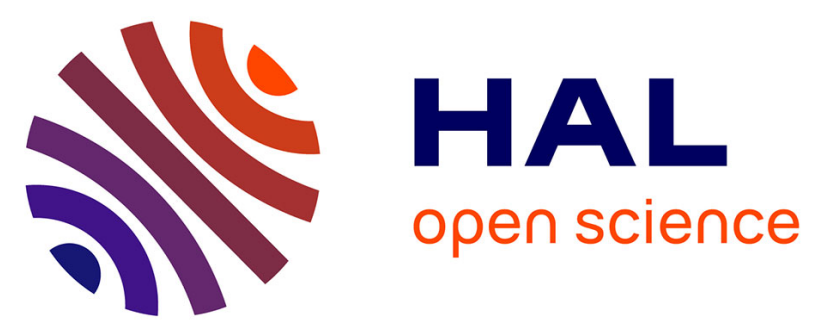

\title{
Development of an international core domain set for medium, large and giant congenital melanocytic naevi as a first step towards a core outcome set for clinical practice and research
}

W Oei, a C Fledderus, P I Spuls, C a M Eggen, J Kottner, C M a M van Der Horst, A Wolkerstorfer, M S Van Kessel, S Krengel, Heather Etchevers, et al.

\section{To cite this version:}

W Oei, a C Fledderus, P I Spuls, C a M Eggen, J Kottner, et al.. Development of an international core domain set for medium, large and giant congenital melanocytic naevi as a first step towards a core outcome set for clinical practice and research. British Journal of Dermatology, 2020, 10.1111/bjd.19694 . hal-03156673

\section{HAL Id: hal-03156673 \\ https://hal-amu.archives-ouvertes.fr/hal-03156673}

Submitted on 2 Mar 2021

HAL is a multi-disciplinary open access archive for the deposit and dissemination of scientific research documents, whether they are published or not. The documents may come from teaching and research institutions in France or abroad, or from public or private research centers.
L'archive ouverte pluridisciplinaire HAL, est destinée au dépôt et à la diffusion de documents scientifiques de niveau recherche, publiés ou non, émanant des établissements d'enseignement et de recherche français ou étrangers, des laboratoires publics ou privés. 


\section{TITLE/ABSTRACT}

Development of an international core domain set for medium, large and giant congenital melanocytic nevi as a first step towards a core outcome set for clinical practice and research

Welling Oei ${ }^{1}$, Phyllis I Spuls ${ }^{2 \pm}$, Anne C Fledderus ${ }^{2,3 \pm}$, Céline A M Eggen ${ }^{1}$, Jan Kottner ${ }^{4}$, Chantal M A M van der Horst ${ }^{3}$ Albert Wolkerstorfer $^{2}$, Marjolein S van Kessel ${ }^{5}$, Sven Krengel ${ }^{6}$, Heather C Etchevers ${ }^{7}$, Ida J Korfage ${ }^{8 *}$, Suzanne G M Pasmans ${ }^{1 *}$

1. Department of Dermatology, Erasmus MC University Medical Center Rotterdam- Sophia Children's Hospital-, Doctor Molewaterplein 40, 3015GD Rotterdam, the Netherlands

2. Department of Dermatology, Amsterdam Public Health, Amsterdam University Medical Center, Meibergdreef 9, 1105 AZ, Amsterdam, the Netherlands

3. Department of Plastic, Reconstructive and Hand Surgery, Amsterdam University Medical Center, University of Amsterdam, Meibergdreef 9, 1105AZ Amsterdam, the Netherlands

4. Charité - Universitätsmedizin Berlin, Charitéplatz 1, D - 10117 Berlin, Germany

5. President Naevus Global, Naevus International Patient Representative Working Group Leader, Utrecht, the Netherlands

6. Dermatological Group Practice, Lindenplatz 6, 23554 Lübeck, Germany

7. Aix Marseille Univ, INSERM, MMG, Faculté de Médecine, 27 boulevard Jean Moulin 13005 Marseille, France

8. Department of Public Health, Erasmus MC, University Medical Center Rotterdam, Doctor Molewaterplein 40, 3015GD Rotterdam, the Netherlands

\pm Shared second author

* Shared last author

Corresponding author during publication: Welling Oei

Department of Dermatology, Erasmus MC University Medical Center Rotterdam- Sophia Children's Hospital,

Wytemaweg 80, $3015 \mathrm{CN}$, Rotterdam, the Netherlands

w.oei@erasmusmc.nl; wellingoei@gmail.com

$+31644780941$

Corresponding author post-publication

S.G.M.A. Pasmans

Department of Dermatology, Erasmus MC University Medical Center Rotterdam- Sophia Children's Hospital,

Wytemaweg 80, $3015 \mathrm{CN}$, Rotterdam, the Netherlands s.pasmans@erasmusmc.nl

Running head (max 70 characters): Core domain set for care and research of congenital melanocytic nevi

This project is funded by the European Academy of Dermatology and Venereology (EADV PPRC-2014-033).

We declare that we have no conflict of interest. 
What's already known about this topic? (max 70 words)

- Medium, large and giant congenital melanocytic nevi (CMN) are associated with psychosocial burden for patients and their family because of their unusual appearance and increased risk of melanoma and/or neurocutaneous melanocytosis.

- Outcome reporting of treatment options for CMN is heterogeneous. Current lack of consensus in outcome reporting hinders the development of evidence-based treatment guidelines for CMN.

- Development of a 'core outcome set' (COS) may enhance standardized reporting.

What does this study add? ( $\max 70$ words)

We focus on the core domain set (CDS), the 'what to measure', for the COS. By following the guidelines of Core Outcome Measures in Effectiveness Trials and the Cochrane Skin Core Outcomes Set Initiative, we reached consensus on six domains, four of which were applied to both care and research: 'quality of life', 'neoplasms', 'nervous system' and 'anatomy of skin'. 'Adverse events' was specific to care and 'pathology' to research.

\section{1b. Abstract (250 words)}

Background: Medium, large and giant congenital melanocytic nevi (CMN) can impose a psychosocial burden on patients and families, and are associated with increased risk of developing melanoma or neurological symptoms. Lack of consensus on what outcomes to measure makes it difficult to advise patients and families about treatment and to set up best practice for CMN. Fostering consensus amongst patient representatives and professionals, we aim to develop a core outcome set (COS), i.e. the minimum set of outcomes to measure and report in care and all clinical trials of a specific health condition. We focused on the "what to measure' aspect, the so-called core domain set (CDS), following COMET and CS-COUSIN guidelines.

Methods: We conducted a systematic review to identify outcomes reported in literature. Focus groups with patient representatives identified patient-reported outcomes. All these outcomes were classified into domains. Through e-Delphi surveys, 144 stakeholders from 27 countries iteratively rated the importance of domains and outcomes. An online consensus meeting attended by seven patient representatives and seven professionals finalized the CDS.

Results: We reached consensus on six domains, four of which were applied to both care and research: 'quality of life', 'neoplasms', 'nervous system' and 'anatomy of skin'. 'Adverse events' was specific to care and 'pathology' to research.

Conclusion: We have developed a CDS for medium-to-giant CMN. Its application in reporting $\mathrm{CMN}$ care and research will facilitate treatment comparisons. The next step will be to reach consensus on the specific outcomes for each of the domains and what instruments should be used to measure these domains/outcomes.

Keywords: Core outcome set, outcome domains, congenital nevi, melanoma, clinical research 


\section{INTRODUCTION}

\section{Background and objectives}

Congenital melanocytic nevi (CMN) are pigmented birthmarks that can cover large areas of the body (1-3). CMN are present at birth or become visible within the first year of life (so-called "tardive" CMN). An estimated $1 \%$ of infants worldwide are born with small CMN. Large and giant $\mathrm{CMN}$ are much rarer, with an estimated incidence of $0.005 \%$ and $0.0002 \%$, respectively (4). CMN are described in size as well as other parameters by the classification system of Krengel et al (5). Medium ( $>1.5 \mathrm{~cm}$ projected adult size [PAS] CMN on the face, and $\geq 10 \mathrm{~cm}$ PAS for the rest of the body), large (> $20 \mathrm{~cm}$ PAS) and giant (>40 $\mathrm{cm}$ PAS) CMN may be associated with a psychosocial burden for patients and their families due to their unusual appearance and the extra medical care CMN may require (6). Large and giant CMN or multiple $\mathrm{CMN}$, are also associated with increased risk of malignant melanoma, soft-tissue tumors or neurologically symptomatic neurocutaneous melanocytosis (1-3, 7-10). Adequate treatment and monitoring of CMN are therefore crucial. Different interventional treatments for CMN such as laser, curettage and excision are available, but conservative treatment such as watchful surveillance is also possible $(3,11-13)$. Patients with CMN may undergo several surgical interventions, sometimes dozens, which do not always yield satisfactory cosmetic and functional results. It is also not established that such treatment reduces the risk of melanoma (3, $12,14)$.

Scientific evidence for the best treatment policies for different kinds of CMN is unfortunately still lacking (15). Multiple case-series articles describe the impact of having CMN, including the effects of treatment, on the lives of patients and their families. However, wide heterogeneity of outcomes reported in these articles makes it difficult to combine, compare or contrast the results (16). Furthermore, outcomes important for patients and patient-reported outcomes are often missing (16). Development of a 'core outcome set' (COS), i.e. the minimum set of outcomes that should be measured and reported in all care settings and clinical trials for a specific health condition, is a potential solution to reduce heterogeneity in outcome selection, measurement, and reporting in future $\mathrm{CMN}$ care and research. This may facilitate evidence synthesis for conservative and interventional treatment recommendations (17).

\section{Scope}

The Outcomes for Congenital Melanocytic Nevi (OCOMEN) project aims to develop a COS for care and research of CMN. A COS consists of 'what' (domains and outcomes) and 'how' to measure (outcome measurement instruments) (18). This article focuses on the development of the core domains sets (CDS) (the 'what to measure').

We aimed to reach consensus on a CDS for care and a separate CDS for research settings. We distinguished the CDS for the care setting, i.e. to be used in every consultation with physicians, as these may or may not be similar to the CDS for the research setting, i.e. to be reported in every research of CMN. We also aimed to initiate the selection of the specific outcomes for each of the domains in the CDS. Fine-tuning the outcomes will be done in a separate project. Such exercises, i.e. fine-tuning the outcomes during the process of reaching consensus on the instruments, have been followed before in other studies (19-21).

We defined a "domain" as an aspect of disease that should be measured, such as cognitive functioning (22), whereas an "outcome" describes a concept or construct which is a part of a domain, such as learning difficulties or memory lapse for the domain "cognitive functioning" (23). 
Specific objectives were:

1. to identify domains and outcomes that

a) have been reported in the literature through a systematic review

b) are considered important by patient representatives, i.e. patients or the parents of minor patients in focus group discussions

2. to compare the domains and outcomes considered critically important by professionals with those of the patient representatives

3. to reach consensus on a CDS of the COS for future use in care and research of CMN,

4. to initiate the selection of the specific outcomes for each domain in the CDS.

We focused on patients with medium, large and giant sizes of $\mathrm{CMN}$ as defined by a recent consensus classification study (5), of any age, without, during, or after treatments.

\section{METHODS}

Our approach was described in a protocol (24). In brief, we followed the guidelines of the Core Outcome Measures in Effectiveness Trials (COMET) initiative (25-27), and the Cochrane SkinCore Outcomes Set Initiative (CS-COUSIN) (28). Results reporting followed the Core Outcome Set-STAndards for Reporting (COS-STAR) (29).

\section{Protocol registration and ethical approval}

This study has been registered on the COMET website (http://www.cometinitiative.org/studies/details/1124) and the CS-COUSIN website (http://cs-cousin.org/cosproject-groups/). Ethical approval was obtained from the Ethical Review Board at the Erasmus MC Rotterdam and from the Ethical Board at the Amsterdam UMC. Prior to their participation, consent was obtained from each of the participants.

\section{Stakeholders recruitment}

We included two English-speaking stakeholder groups from 27 countries: one of patient representatives and one of professionals. We reached out to patient representatives through patient advocacy groups, hospital registries in the Netherlands, publicity through Naevus International (https://www.naevusinternational.com/2018/09/26/the-outcome-measures-forcongenital-melanocytic-naevi-ocomen-project/) and at its meetings in 2017 and 2018, word-ofmouth and social media. We involved professionals from various backgrounds (dermatologists, plastic surgeons, pediatricians, psychologists, pathologists, and basic researchers). Invitations were sent to all patient representatives and professionals who had earlier expressed interest in participation. Detailed recruitment is described in the protocol (24).

In the development of the CDS, we were supported by a study advisory group. This group consisted of experts in COS development and in the field of CMN, including a patient representative, researchers, dermatologists and a plastic surgeon. The study advisory group provided input considering protocol development, stakeholder recruitment, design of the eDelphi, and the consensus meeting.

\section{Identifying an initial list of outcomes}

Our methods in identifying the initial list of outcomes have been described in the protocol (24). Briefly, we listed the outcomes from systematic review, focus group discussions and guidelines (Figure 1). We looked at a list of outcomes in the taxonomy published by the COMET initiative 
website $(22,26)$ to ensure that we did not miss any relevant outcomes that were not reported by the review or focus groups. Several domains of the COMET taxonomy that were generally irrelevant for our aim, such as cardiac, ear and labyrinth, or hepatobiliary outcomes, were excluded. Since CMN is a specific skin malformation, and the COMET taxonomy provides a high-level classification covering various diseases, we also consulted the WHO International Classification of Functioning, Disability and Health (ICF) for a more detailed classification of skin anatomy and functions (30).

An overview of the selection of domains and outcomes is summarized in Figure 1.

\section{Consensus process: e-Delphi}

Delphi surveys are a well-recognized and increasingly used consensus method for COS development (31-33). We conducted three rounds of e-Delphi, using the Lime Survey platform. Participants were given three weeks to complete a survey. Reminders were sent frequently after the first week. The ratings from the two stakeholder groups (patient representatives and professionals) were evaluated separately. During the first two rounds, we also recorded characteristics of the patient CMN associated with the representatives, including size, color, location, and treatment history. For the professional group, we recorded the type of profession and years of experience.

In the first round, participants from the two groups were asked to rate the importance of domains for clinical care and for the research setting separately. During the first two rounds, participants could suggest additional domains. The results of the previous round were fed back to the participants. In the second round, participants could alter their votes. In the third round, participants were only asked to vote again for those domains for which no consensus had been reached. Moreover, in the third round, participants were asked to also rate the list of outcomes as proposed for each domain.

The consensus definitions for the domains and outcomes are specified in the protocol. Briefly, domains were rated on 9-point Likert scales, where 1 signifies no importance and 9 very important. Domains scored 7, 8, or 9 by at least $70 \%$ of one of the stakeholder groups were considered to be 'important domains' for that stakeholder group and domains that were scored 1,2 or 3 by at least $70 \%$ of one stakeholder group were considered 'unimportant domains'. Domains that were considered 'important domains' by both stakeholder groups, were considered to have met the consensus definition and were eligible for provisional inclusion in a CDS and named 'provisional consensus domains'. Domains that were considered 'unimportant domains' by both stakeholder groups, were dropped.

For the outcomes that describe the domains, those that were selected by at least $70 \%$ of participants of the third round of the e-Delphi were considered to be 'important outcomes' and eligible for a vote for inclusion in the CDS during the consensus meeting.

\section{Consensus process: consensus meeting}

An online consensus meeting involving the study advisory group and representatives of eDelphi completers was planned to review and discuss the 'provisional consensus domains' from the e-Delphi surveys. Patient representatives and professionals were intentionally sampled in equal proportion from the e-Delphi completers to ensure representation of patient representatives with various geographical backgrounds as well as types of profession (dermatologists, surgeons, pathologists, researchers). This intentional sampling deviated from the protocol. 
During the meeting, participants discussed and voted on the domains for which the abovementioned consensus definition was reached, and on domains that were considered important by at least a stakeholder group during the e-Delphi process, for inclusion in the CDS, separately for care and for research settings. The importance of domains was voted on during the online consensus meeting. Participants filled out the voting form for each domain, and the forms were sent to the organizer directly. The responses were calculated immediately and fed back to the participants. After a break of 15 minutes, we used this vote result to deliberate and narrow down the top 5 domains through another direct voting session by email. Several studies recommended to be very selective about the number of consensual domains to be included in the CDS, to promote feasibility and future uptake $(26,34)$. The average (median) number of domains reported in COS studies was five (26). Therefore, participants were asked to also select their top five from the list of 'provisional consensus domains' for the CDS. Moreover, participants had the opportunity to discuss the classifications of domains and could make suggestions to lump or split the domains.

Besides the rating of domains, participants were asked to vote on those shortlisted outcomes considered 'important' during the last e-Delphi round for inclusion in the CDS. Outcomes that received favorable votes from at least $70 \%$ of meeting participants were included in the CDS. For practical reasons, the outcome voting was done by e-mail.

\section{Statistical analyses}

Descriptive statistics were used to summarize the results of each round, including the percentage of stakeholder groups who had given a high score $(7,8$, or 9$)$ per domain. The analysis was done in $\mathrm{R}$, version 3.5.1.

\section{RESULTS}

\section{Identifying an initial list of outcomes}

An initial list of 123 outcomes reported in the literature of CMN was found in a systematic review(16). Through seven focus group discussions (five in the Netherlands and two international), we identified 38 additional outcomes that are important for patient representatives (24). From the COMET and the ICF we obtained additional 20 outcomes. After removal of redundancies ( 2 outcomes), we grouped the 179 outcomes into domains by following the COMET taxonomy $(22,26)$. The selected domains (30) and outcomes (179) were fed into the e-Delphi study (Appendix 1- List of domains and outcomes for the e-Delphi).

\section{Delphi survey}

In total, we identified 138 professionals and 134 patient representatives. Among those, 186 confirmed their interest in participation by mail and thus, received the invitation link to access the first survey. The response rate and the characteristics of participants are presented in Table 1. In each round, around $70 \%$ of participants completed the e-Delphi. The median age of participants is 43 years old (parents of young patients filled out the e-Delphi on behalf of their children) and around $70 \%$ of the participants are female. There was equal representation of patient representatives and professionals. The geographical spread of the participants from 27 countries on six continents has been described elsewhere (24).

During the first round three domains (itch, sweating, overheating) were suggested to be added by participants but were not rated as sufficiently important in the subsequent rounds. 
To give an overview of the rating of all domains, Figure 2 summarizes the rating of the complete list of domains during the second round of the e-Delphi. The rating during this second round showed that the preferences of patient representatives and of professionals on important domains for care were rather similar to that for the research setting, with a few exceptions such as family function or pain. After the second round, seven 'provisional consensus domains' were eligible for inclusion in the CDS.

Table 2 summarizes the rating of 'important domains', i.e., domains that had ever scored 7, 8, or 9 by at least $70 \%$ of a stakeholder group, for a particular setting (care or research), during the three rounds of e-Delphi. No domains were voted to be 'unimportant' during the three rounds of e-Delphi. It appeared that the preferences of patient representatives and professionals for the 'important' domains were consistent throughout the e-Delphi rounds. Despite the few changes in preference of domains such as cognitive functions, social function and psychiatric outcomes, most stakeholders agreed on the importance of the selected domains.

After the third round, there were 15 'provisional consensus domains' eligible for inclusion in the CDS for the care and 9 for the research setting (Table 3), with quite some overlap. In total, there were 16 unique provisional consensus domains. For those 16 unique domains, 45 outcomes were selected by at least $70 \%$ of stakeholder groups. A summary of results of the first, second, and third e-Delphi rounds can be found in Appendix 2, Appendix 3, and Appendix 4, respectively.

\section{Consensus meeting}

Detailed descriptions of the consensus meeting can be found in Appendix 5. Seventeen people participated in the consensus meeting, including seven patient representatives and seven professionals, and three (no vote) project members. Fourteen participants reviewed and voted on the 'provisional consensus domains' for inclusion in the CDS (Table 3). They also voted on the 'important domains' for which consensus had not been reached during the eDelphi (Appendix 5-Table A2A and Table A2B); none of these 'important domains' were added to the 'provisional consensus domains'. During the discussion, re-classifications of a number of domains were suggested by either lumping or splitting domains. Since it was unanimously agreed to lump the 'social', 'family', 'emotional', and 'physical function' into a new domain called 'quality of life', this idea was immediately implemented. Other suggestions included categorization of 'death', 'neoplasms', 'nervous system', and '(histo)pathology' into the domain 'malignancy'; and 'signs/symptoms' into the domain 'adverse events'. These suggestions were not unanimously accepted and thus not included. Furthermore, the domain 'histopathology' was renamed as 'pathology' because 'pathology' does not only describe histological findings but also molecular findings. The selection of the top five domains for the CDS and the opportunity to alter the classification of domains was an additional step to promote future uptake, and had not been specified in the initial protocol (24).

The top five domains that were considered crucial for care and for the research setting are shown schematically in Table 4 and Figure 3. The core domains of the COS for CMN comprise the categories 'quality of life', 'neoplasms', 'nervous system', and 'anatomy of skin' for both the care and research settings, and the domains 'general adverse events' or 'pathology' for clinical care or research, respectively. There was a thorough discussion on whether or not general adverse events should be included in the COS for the research setting, given it was considered crucial but did not get sufficient votes to be among the top five domains. There was a concern that if we were to make an exception here, it would be hard to draw the line for other domains that were also not included in the COS. Therefore, we decided 


\section{to follow the consensus meeting protocol sent to participants prior to the meeting, in which it was stated that we would include only the top five domains for each setting.}

For the domains included in the CDS, 44 outcomes describing these domains were considered to be 'important by stakeholders during the third e-Delphi round. During the consensus meeting, fourteen of the seventeen participants re-voted for these outcomes. Table 5 presents a list of 28 outcomes that were selected by at least $70 \%$ of the consensus meeting participants for inclusion in the CDS. One outcome, 'brain complications', was re-classified from the domain 'general adverse events' to the domain 'nervous system' since they may not be related to treatment.

\section{DISCUSSION}

Our project presents the first part of the COS development for medium, large and giant CMN that considers the opinions of patient representatives and uses transparent methods planned $a$ priori. The purpose of this study was to develop an international consensus-based CDS to be applied in care of or in research about medium-to-giant CMN patients of any age, with or without treatment. We managed to include participation from six continents.

To date, there is no outcome classification specifically designed for skin conditions (35). Several COS studies on skin diseases set examples of outcome classification $(20,34,36,37)$. We chose to enrich the COMET domain classification by adding the classification from the WHO ICF for skin anatomy and functions. Domains that were classified as 'Functioning' in the COMET taxonomy were grouped into 'quality of life' during our consensus meeting.

Participants frequently brought up the importance of providing vetted information about CMN throughout the focus group discussions and it was rated highly during the e-Delphi process. However, during the consensus meeting, information provision was removed from the 'provisional consensus domains' since it was not considered to be directly influenced by either conservative or interventional treatment.

The consensus meeting took place online to allow participation of people around the globe. We successfully reached consensus on many domains within a limited time of online discussion. It does, however, require great concentration to participate in an online discussion in a non-native language, time zones were not equally convenient for all, and participants did not always have a stable internet connection, which can interrupt the discussion. Nevertheless, we found that the online consensus meeting provided a complementary opportunity to the surveys to directly discuss, debate and finally agree on the important domains.

In COS development, adoption of an internationally accepted outcome domain classification is supposed to facilitate literature searches and synthesis of evidence (38). The COMET taxonomy $(25,26)$ that we chose to follow is open to more detailed classification relative to clinical and methodological areas (26). Too much detail, however, might cause confusion when one needs to formulate specifically what a domain should entail. We attempted to identify which outcomes should be considered when looking at each of the core domains through two selection processes, i.e., during the last e-Delphi and by email after the consensus meeting. Some outcomes were classified under one domain but remained related to another. Moreover, participants did not have the opportunity to vote separately for the specific outcomes they considered important. Therefore, the selected outcomes in this study were used to illustrate what a core domain could consist of. To facilitate further consensus on finding or 
developing the instruments to measure these outcomes and to promote future uptake, it may be necessary to reorganize or reduce the number of outcomes in each setting (39).

There were some limitations of the COS development in this study. Participation from North America and Europe was over-represented in our study. In part, this might be due to the requirement to conduct the development process online and in English. The essential votes included 14 intentionally selected participants, in contrast to the random sampling stated in the protocol. We thought that equal representation of patient representatives and professionals was essential, but there were more professionals who were available to participate in the consensus meeting. Furthermore, "general adverse events" was excluded from the CDS of research and "delivery of care" from the CDS for the clinical setting after the consensus meeting. These findings may be contested by participants who had voted for these domains by e-mail but could not participate in the consensus meeting. We have sent these interim results to all participants who were involved in the second round of the e-Delphi, and none has objected to the CDS selected by the online consensus meeting participants.

In conclusion, the present study reports the development of a comprehensive CDS for use in care and in research on medium-to-giant CMN. The final CDS includes the top five domains for care and top five for research, with four in common. A set of outcomes was selected to illustrate these domains. Future research is needed to reach a consensus on the core outcomes of each domain in the CDS, to validate and/or to develop instruments, and to reach a consensus on the appropriate outcome measurement instruments. Uptake of this CDS in future clinical care of, and research about medium-to-giant $\mathrm{CMN}$ should facilitate comparisons across different treatment choices for both patients and professionals.

\section{Acknowledgements}

This project is part of the European Reference Network-SKIN thematic group Cutaneous Mosaic Disorders : - Nevi \& Naevoid skin disorders - Complex vascular malformations and vascular tumors (https://ern-skin.eu/). This project was supported partly by a grant from the European Academy of Dermatology and Venereology.

HCE was supported by funding from the Association du Naevus Géant Congénital, Naevus 2000 France-Europe and the Asociación Española de Nevus Gigante Congénito.

\section{List of Appendix}

Appendix 1- List of domains and outcomes for the e-Delphi

Appendix 2- Summary of the first e-Delphi

Appendix 3- Summary of the second e-Delphi

Appendix 4- Summary of the third e-Delphi

Appendix 5- Summary of the consensus meeting

\section{REFERENCES}

1. Egan $\mathrm{CL}$, Oliveria SA, Elenitsas $\mathrm{R}$ et al. Cutaneous melanoma risk and phenotypic changes in large congenital nevi: a follow-up study of 46 patients. Journal of the American Academy of Dermatology. 1998;39(6):923-32.

2. Kadonaga JN, Frieden IJ. Neurocutaneous melanosis: definition and review of the literature. Journal of the American Academy of Dermatology. 1991;24(5 Pt 1):747-55. 
3. Marghoob AA, Agero AL, Benvenuto-Andrade C, Dusza SW. Large congenital melanocytic nevi, risk of cutaneous melanoma, and prophylactic surgery. Journal of the American Academy of Dermatology. 2006;54(5):868-70; discussion 71-3.

4. Hamm H, Hoger PH. Skin tumors in childhood. Deutsches Arzteblatt International. 2011;108(20):347-53.

5. Krengel S, Scope A, Dusza SW et al. New recommendations for the categorization of cutaneous features of congenital melanocytic nevi. Journal of the American Academy of Dermatology. 2013;68(3):441-51.

6. Koot HM, de Waard-van der Spek F, Peer CD et al. Psychosocial sequelae in 29 children with giant congenital melanocytic naevi. Clinical and experimental dermatology. 2000;25(8):589-93.

7. Etchevers HC. Hiding in plain sight: molecular genetics applied to giant congenital melanocytic nevi. Journal of Investigative Dermatology. 2014;134(4):879-82.

8. Krengel S, Hauschild A, Schafer T. Melanoma risk in congenital melanocytic naevi: a systematic review. The British journal of dermatology. 2006;155(1):1-8.

9. Watt AJ, Kotsis SV, Chung KC. Risk of melanoma arising in large congenital melanocytic nevi: a systematic review. Plastic and reconstructive surgery. 2004;113(7):1968-74.

10. Bittencourt FV, Marghoob AA, Kopf AW et al. Large congenital melanocytic nevi and the risk for development of malignant melanoma and neurocutaneous melanocytosis. Pediatrics. 2000;106(4):73641.

11. Pasmans S, Eggen C., Bergman W et al. Multidisiplinaire Richtlijn Congenitale Melanocytaire Naevi 2017.

12. Ibrahimi OA, Alikhan A, Eisen DB. Congenital melanocytic nevi: where are we now? Part II. Treatment options and approach to treatment. Journal of the American Academy of Dermatology. 2012;67(4):515 e1-13; quiz 28-30.

13. Krengel S, Marghoob AA. Current management approaches for congenital melanocytic nevi. Dermatol Clinics. 2012;30(3):377-87.

14. Price HN, Schaffer JV. Congenital melanocytic nevi-when to worry and how to treat: Facts and controversies. Clinics in Dermatology. 2010;28(3):293-302.

15. Kinsler VA, Reyes-Mugica M, Marghoob A. Congenital Melanocytic Naevi. In: Fisher DE, Bastian BC, editors. Melanoma. New York, NY: Springer New York; 2019. p. 1-30.

16. Fledderus A, Franke $C$, Eggen $C$ et al. Outcomes and measurement instruments used in Congenital Melanocytic Naevi research: A systematic review. Journal of Plastic, Reconstructive \& Aesthetic Surgery. 2019.

17. Williamson PR, Altman DG, Blazeby JM et al. Developing core outcome sets for clinical trials: issues to consider. Trials [Electronic Resource]. 2012;13:132.

18. Kirkham JJ, Davis K. Core Outcome Set-STAndards for Development: The COS-STAD recommendations. 2017;14(11):e1002447.

19. Chalmers J, Schmitt J, Apfelbacher $\mathrm{C}$ et al. Report from the third international consensus meeting to harmonise core outcome measures for atopic eczema/dermatitis clinical trials (HOME). The British journal of dermatology. 2014;171(6):1318-25.

20. Horbach SER, van der Horst C, Blei F et al. Development of an international core outcome set for peripheral vascular malformations: the OVAMA project. The British journal of dermatology. 2018;178(2):473-81.

21. Schmitt J, Langan S, Stamm T, Williams HC, panel HOMiED. Core outcome domains for controlled trials and clinical recordkeeping in eczema: international multiperspective Delphi consensus process. Journal of Investigative Dermatology. 2011;131(3):623-30.

22. COMET inititiative. Outcome classification taxonomy with examples table. 2018.

23. Prinsen CA, Vohra S, Rose MR et al. Core Outcome Measures in Effectiveness Trials (COMET) initiative: protocol for an international Delphi study to achieve consensus on how to select outcome measurement instruments for outcomes included in a 'core outcome set'. Trials [Electronic Resource]. 2014;15:247. 
24. Oei W, Fledderus AC, Korfage I et al. Protocol for the development of core set of domains of the core outcome set for patients with congenital melanocytic naevi (OCOMEN project). Journal of the European Academy of Dermatology and Venereology. 2019.

25. Williamson PR, Altman DG, Bagley $\mathrm{H}$ et al. The COMET Handbook: version 1.0. Trials [Electronic Resource]. 2017;18(Suppl 3):280.

26. Dodd S, Clarke M, Becker L et al. A taxonomy has been developed for outcomes in medical research to help improve knowledge discovery. Journal of clinical epidemiology. 2018;96:84-92.

27. Kirkham JJ, Davis K, Altman DG et al. Core Outcome Set-STAndards for Development: The COSSTAD recommendations. PLoS Medicine / Public Library of Science. 2017;14(11):e1002447.

28. Schmitt J, Deckert S, Alam M et al. Report from the kick-off meeting of the Cochrane Skin Group Core Outcome Set Initiative (CSG-COUSIN). Brit J Dermatol. 2016;174(2):287-95.

29. Kirkham JJ, Gorst S, Altman DG et al. Core Outcome Set-STAndards for Reporting: The COSSTAR Statement. PLoS Medicine / Public Library of Science. 2016;13(10):e1002148.

30. Organization WH. WHO International Classification of Functioning, Disability and Health (ICF) [Internet]26-10-2019 [cited 26-10-2019]. Podcast. Available from:

http://apps.who.int/classifications/icfbrowser/

31. Sinha IP, Smyth RL, Williamson PR. Using the Delphi Technique to Determine Which Outcomes to Measure in Clinical Trials: Recommendations for the Future Based on a Systematic Review of Existing Studies. Plos Medicine. 2011;8(1).

32. Williams PL, Webb C. The Delphi technique: a methodological discussion. The Journal of Advanced Nursing. 1994;19(1):180-6.

33. Hasson F, Keeney S, McKenna H. Research guidelines for the Delphi survey technique. J Adv Nurs. 2000;32(4):1008-15.

34. Schmitt J, Apfelbacher C, Spuls PI et al. The Harmonizing Outcome Measures for Eczema (HOME) Roadmap: A Methodological Framework to Develop Core Sets of Outcome Measurements in Dermatology. Journal of Investigative Dermatology. 2015;135(1):24-30.

35. Kottner J, Schmitt J. Core outcome set in dermatology: next steps. The British journal of dermatology. 2018;179:549-50.

36. Kottner J, Jacobi L, Hahnel E et al. Core outcome sets in dermatology: report from the second meeting of the International Cochrane Skin Group Core Outcome Set Initiative. Brit J Dermatol. 2018;178(4):e279-e85.

37. van Geel N, Lommerts JE, Bekkenk MW et al. Development and validation of a patient-reported outcome measure in vitiligo: The Self Assessment Vitiligo Extent Score (SA-VES). Journal of the American Academy of Dermatology. 2017;76(3):464-71.

38. Williamson P, Clarke M. The COMET (Core Outcome Measures in Effectiveness Trials) Initiative: Its Role in Improving Cochrane Reviews. Cochrane Database of Systematic Reviews. 2012(5):ED000041.

39. Schmitt J, Lange T, Kottner J et al. Cochrane reviews and dermatological trials outcome concordance: why Core Outcome Sets could make trial results more usable. Journal of Investigative Dermatology. 2019;139(5):1045-53. 
Table 1- Summary of responses to the e-Delphi surveys

\begin{tabular}{llll}
\hline & $\begin{array}{l}\text { First round } \\
\mathrm{n}=186\end{array}$ & $\begin{array}{l}\text { Second round } \\
\mathrm{n}=144\end{array}$ & $\begin{array}{l}\text { Third round } \\
\mathrm{n}=111\end{array}$ \\
\hline $\begin{array}{l}\text { Responses }(\%) \\
\text { Stakeholder group }\end{array}$ & $144(77)$ & $111(77)$ & $70(63)$ \\
$\quad \begin{array}{l}\text { Patients representatives }(\%) * * \\
\text { Professionals (\%)** }\end{array}$ & $81(56)$ & $62(56)$ & $32(46)$ \\
* & $63(44)$ & $49(44)$ & $38(54)$ \\
\hline
\end{tabular}

percentage indicates proportion of responders of a round among those invited (n) for that round.

** percentage indicates proportion of a stakeholder group among those responders.

Table 2- Percentage of score 7, 8, or 9 given by patient representatives (PP) and professionals (PRO) on domains for clinical care or for the research setting during each eDelphi round

\begin{tabular}{|c|c|c|c|c|c|c|c|c|c|c|c|c|c|}
\hline \multirow[t]{3}{*}{ No } & \multirow{3}{*}{$\begin{array}{l}\text { Domains that are scored as } \\
\text { important by a stakeholder } \\
\text { group }\end{array}$} & \multicolumn{4}{|c|}{ First round } & \multicolumn{4}{|c|}{ Second round } & \multicolumn{4}{|c|}{ Third round } \\
\hline & & \multicolumn{2}{|c|}{ Care } & \multicolumn{2}{|c|}{ Research } & \multicolumn{2}{|c|}{ Care } & \multicolumn{2}{|c|}{ Research } & \multicolumn{2}{|c|}{ Care } & \multicolumn{2}{|c|}{ Research } \\
\hline & & PP & $\begin{array}{c}\text { PR } \\
\mathrm{O}\end{array}$ & PP & $\begin{array}{c}\text { PR } \\
\text { O }\end{array}$ & PP & $\begin{array}{c}\text { PR } \\
\text { O }\end{array}$ & PP & $\begin{array}{c}\text { PR } \\
\mathrm{O}\end{array}$ & PP & $\begin{array}{c}\text { PR } \\
\text { O }\end{array}$ & PP & $\begin{array}{c}\text { PR } \\
\mathrm{O}\end{array}$ \\
\hline 1 & Delivery of care & 73 & 83 & 68 & 73 & 85 & 94 & 73 & 88 & $*$ & $*$ & $*$ & * \\
\hline 2 & Cognitive function & 41 & 53 & 62 & 41 & 57 & 50 & 67 & 53 & 74 & 44 & 71 & 36 \\
\hline 3 & Emotional function & 86 & 87 & 75 & 75 & 90 & 88 & 71 & 75 & * & $*$ & $*$ & $*$ \\
\hline 4 & Family function & 73 & 83 & 68 & 73 & 78 & 77 & 60 & 52 & * & $*$ & 62 & 44 \\
\hline 5 & Social function & 70 & 76 & 67 & 60 & 96 & 80 & 71 & 60 & * & * & 55 & 53 \\
\hline 6 & Neoplasms & 83 & 90 & 85 & 87 & 89 & 98 & 89 & 98 & * & * & $*$ & * \\
\hline 7 & Nervous system & 83 & 86 & 85 & 79 & 84 & 90 & 83 & 87 & * & * & $*$ & * \\
\hline 8 & Psychiatric outcomes & 72 & 60 & 65 & 52 & 76 & 60 & 78 & 44 & 85 & 71 & 76 & 53 \\
\hline 9 & Pain & 79 & 81 & 72 & 63 & 81 & 86 & 70 & 67 & * & * & 81 & 71 \\
\hline 10 & $\begin{array}{l}\text { Anatomy characteristics of } \\
\text { skin }\end{array}$ & 90 & 94 & 89 & 84 & 87 & 90 & 92 & 86 & * & * & $*$ & * \\
\hline 11 & $\begin{array}{l}\text { Anatomy characteristics of } \\
\text { hair }\end{array}$ & 71 & 78 & 78 & 62 & 76 & 67 & 71 & 52 & 57 & 44 & 57 & 24 \\
\hline 12 & Histopathology & 83 & 73 & 84 & 86 & 71 & 63 & 87 & 75 & 69 & 84 & $*$ & $*$ \\
\hline 13 & Protective function & 75 & 67 & 74 & 51 & 76 & 61 & 79 & 40 & 76 & 58 & 33 & 62 \\
\hline 14 & Repair function & 81 & 68 & 81 & 51 & 75 & 71 & 79 & 44 & * & * & 74 & 53 \\
\hline 15 & Sensation of skin & 78 & 59 & 77 & 44 & 81 & 65 & 75 & 38 & 76 & 49 & 67 & 31 \\
\hline 16 & Other function & 67 & 38 & 77 & 44 & 46 & 17 & 40 & 13 & 36 & 16 & 36 & 11 \\
\hline 17 & General adverse events & 79 & 84 & 77 & 75 & 84 & 94 & 81 & 79 & * & * & $*$ & * \\
\hline 18 & Infection & 79 & 67 & 69 & 52 & 89 & 67 & 76 & 44 & 90 & 82 & 76 & 49 \\
\hline 19 & Death & 80 & 86 & 83 & 83 & 82 & 94 & 90 & 94 & * & * & $*$ & * \\
\hline 20 & Information provision & 91 & 81 & 77 & 60 & 92 & 86 & 78 & 52 & * & $*$ & 79 & 51 \\
\hline
\end{tabular}


Table 3- List of 'provisional consensus domain' (X) from the e-Delphi for inclusion in the CDS during the consensus meeting.

\begin{tabular}{|l|c|c|}
\hline Domains & Care & Research \\
\hline 1. Delivery of care & $\mathrm{X}$ & $\mathrm{X}$ \\
2. Emotional function & $\mathrm{X}$ & $\mathrm{X}$ \\
3. Social function & $\mathrm{X}$ & \\
4. Family function & $\mathrm{X}$ & \\
5. Physical functioning & $\mathrm{X}$ & \\
6. Malignancy (neoplasms) & $\mathrm{X}$ & $\mathrm{X}$ \\
7. Nervous system & $\mathrm{X}$ & $\mathrm{X}$ \\
8. Psychiatric outcomes & $\mathrm{X}$ & \\
9. Pain & $\mathrm{X}$ & $\mathrm{X}$ \\
10. Anatomy of skin & $\mathrm{X}$ \\
11. (Histo)pathology & $\mathrm{X}$ & $\mathrm{X}$ \\
12. Repair function of skin & $\mathrm{X}$ & $\mathrm{X}$ \\
13. General adverse events & $\mathrm{X}$ & \\
14. Infection & $\mathrm{X}$ & $\mathrm{X}$ \\
15. Death & & \\
16. Information provision & & \\
\hline
\end{tabular}

Table 4- Results of vote on the provisional consensus domains; The top 5 domains (highlighted) were included in the CDS for the care or research setting.

\begin{tabular}{|l|l|c|c|l|c|}
\hline No & Domains for the care setting & $\begin{array}{l}\text { Vote } \\
\%\end{array}$ & No & Domain for the research setting & $\begin{array}{l}\text { Vote } \\
\%\end{array}$ \\
\hline 1 & $\begin{array}{l}\text { Quality of life (including } \\
\text { social, family, emotional and } \\
\text { physical function) }\end{array}$ & 86 & 1 & Pathology & 93 \\
\hline 2 & Neoplasms & 79 & 2 & Neoplasms & 79 \\
\hline 3 & Nervous system & 64 & 3 & Nervous system & 71 \\
\hline 4 & Anatomy of skin & 71 & 4 & $\begin{array}{l}\text { Quality of life (emotional } \\
\text { function) }\end{array}$ & 71 \\
\hline 5 & General adverse events & 57 & 5 & Anatomy of skin & 64 \\
\hline 6 & Delivery of care & 50 & 6 & General adverse events & 43 \\
\hline 7 & Infection & 21 & 7 & Death & 43 \\
\hline 8 & Pain & 21 & 8 & Pain & 14 \\
\hline 9 & Death & 14 & 9 & Delivery of care & \\
\cline { 1 - 2 } 10 & Psychiatric outcomes & 14 & & & \\
\cline { 1 - 2 } 11 & Repair function & 7 & & &
\end{tabular}


Table 5- List of domains and outcomes selected for the COS

\begin{tabular}{|c|c|}
\hline Domains & Outcomes \\
\hline 1. Quality of life* & $\begin{array}{l}\text { 1. Acceptance of CMN as part of identity } \\
\text { 2. Satisfaction with treatment choice } \\
\text { 3. Coping mechanisms } \\
\text { 4. Esthetic issues } \\
\text { 5. Perceived stigmatization } \\
\text { 6. Social relations* } \\
\text { 7. Acceptance by parents/family members of having } \mathrm{CMN}^{*}\end{array}$ \\
\hline 2.Neoplasms/malignancy & $\begin{array}{l}\text { 8. Incidence of melanoma } \\
\text { 9. Incidence of other malignancy } \\
\text { 10. Frequency of monitoring for malignancy } \\
\text { 11. Biopsy findings/ histological characteristics }\end{array}$ \\
\hline 3. Nervous system & $\begin{array}{l}\text { 12. Epilepsy } \\
\text { 13. MRI findings } \\
\text { 14. Hydrocephalus } \\
\text { 15. Motor development } \\
\text { 16. Brain complications due to melanocytosis, melanoma, or } \\
\text { metastasis }\end{array}$ \\
\hline $\begin{array}{l}\text { 4. Anatomical } \\
\text { characteristics of skin }\end{array}$ & $\begin{array}{l}\text { 17. Color of the CMN (hypo-, hyperpigmentation, vitiligo) } \\
\text { 18. Hairiness } \\
\text { 19. Lumpiness } \\
\text { 20. Spontaneous regression of nevi } \\
\text { 21. New satellite nevi } \\
\text { 22. Change of nevus over time } \\
\text { 23. Rugosity }\end{array}$ \\
\hline 5. General adverse events $\$$ & $\begin{array}{l}\text { 24. Growth-related problem in the area of operated nevus } \\
\text { 25. Skin graft issues (flap, graft failure) } \\
\text { 26. Change in scar (keloid, hypertrophic, atrophic, widening, } \\
\text { contracture) } \\
\text { 27. Cranial or facial deformation by treatment }\end{array}$ \\
\hline 6. Pathology \& & 28. Histo-pathological characteristics \\
\hline
\end{tabular}

* Quality of life entails emotional, physical, family, and social functioning for the care setting where as for the research setting it entails only emotional functioning.

$\$$ General adverse events was chosen by vote within the care setting only.

${ }^{\&}$ Pathology was chosen by vote within the research setting only. 


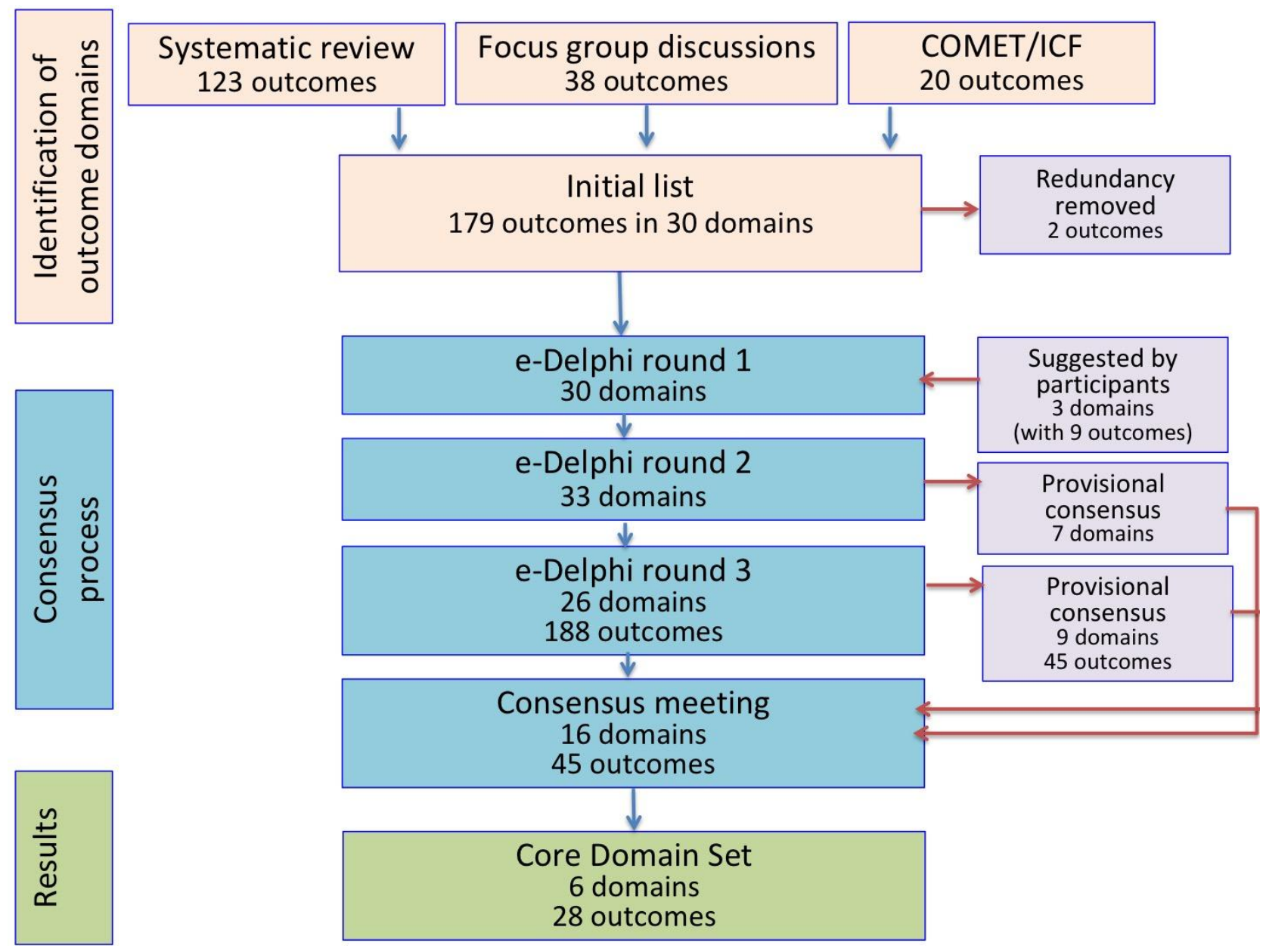

Figure 1- Overview of CDS development 

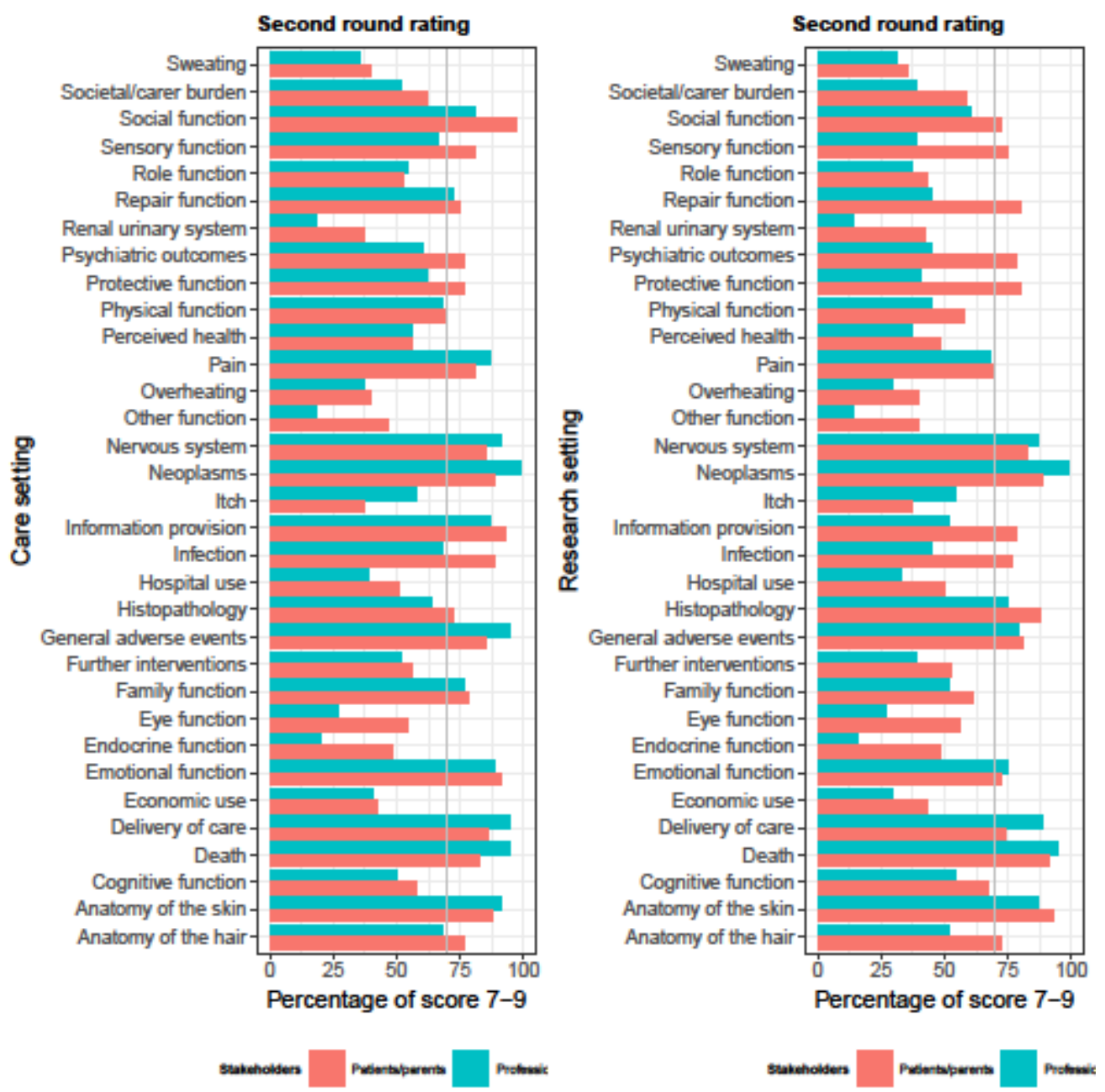

Figure 2- The proportion of patient representatives (red bars) or professionals (blue bars) who assigned a score of 7, 8 or 9 to each of the domains for care (left) and for the research (right) settings during the second round of e-Delphi. 


\section{Core domain set}

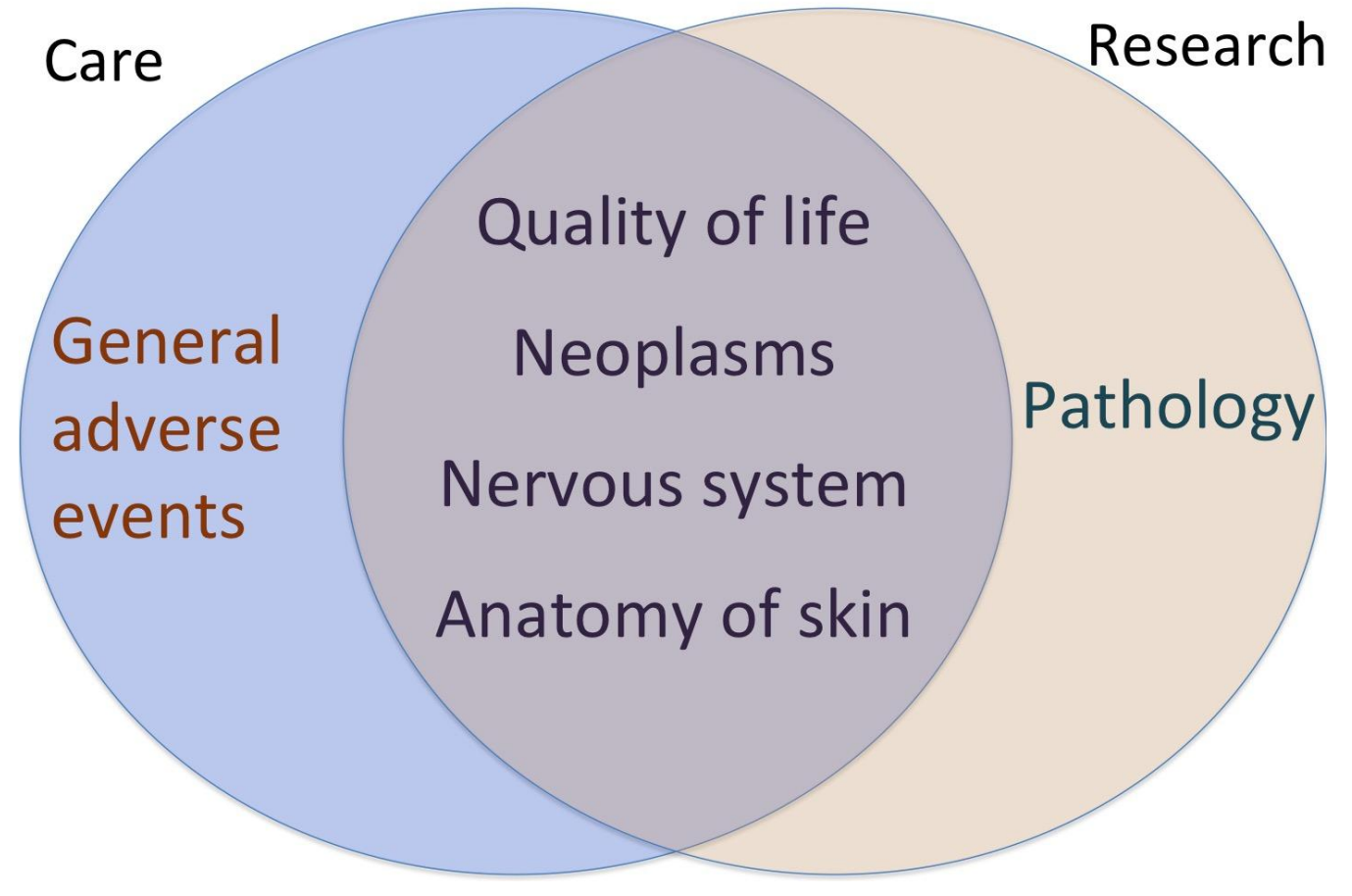

Figure 3- Core domains of the COS for reporting clinical care of CMN and research into CMN. 